\title{
Hormone Replacement Therapy: An Update
}

\author{
Khadilkar Suvarna
}

Published online: 21 August 2012

(C) Federation of Obstetric \& Gynecological Societies of India 2012

Ever since menopause was causally linked to several symptoms and disease processes, the concept of hormone replacement therapy (HRT) took root and was widely practiced over half a century. We have witnessed the rise and fall of the HRT during these years. While the life expectancy has improved enormously, the average age of menopause has not changed, and hence a vast majority of women spend one third of their lives in the state of estrogen deficiency. The long-term problems of menopause, therefore, are important to the present times.

It is rather interesting to study the changing trends in hormonal management of menopause. There was a time when menopause was managed without hormones because of lack of knowledge. This was followed by selective use of HRT and then the wide and universal use of HRT, only to realize the emergence of health risks related to HRT. Hence, the approach had to be changed again. After having gone through these changes, we seem to have completed the cycle and stand at a point not far away from where we began, but with a lot of knowledge and conclusions.

Khadilkar S., Professor and HOD

Department of Obstetrics and Gynecology, RCSMGMC, Kolhapur, India

Khadilkar S. $(\bowtie)$, Professor and HOD

61/62 New Clement Court, Off Gokhale Road [North], Opposite Amar Hind Mandal, Shivaji Park, Dadar, Mumbai 400028, India e-mail: suvarnakhadilkar@yahoo.com
The main pathbreaking trial has been the Women's Health initiative (WHI) [1] trial in the year 2002. It has changed clinical practice and has led us toward a more rational interpretation of the place of hormone therapy in menopause management.

\section{The WHI Trial}

The WHI trial enrolled approximately 16,000 women nationwide, from the ages of 50-79 years. The average age of women in the study was 63 years. The major goal of the WHI clinical trial was to determine whether combined estrogen and progestin HRT prevented heart disease and fractures, and whether there were associated risks. After an average of 5 years of follow-up, heart disease and stroke were significantly increased in HRT users by 29 and $41 \%$, respectively. Venous thromboembolic events (VTEs) were increased twofold. Approximately 11,000 women without a uterus participated in a separate WHI study [2] and were randomized either to estrogen alone or to placebo. After an average follow-up of 7 years, there was no increased risk of heart disease in estrogen users. Estrogen use did have adverse vascular effects, increasing the risk of stroke by $39 \%$ and VTEs by $33 \%$.

However, the harmful findings in the older women in both HRT trials tended to be extrapolated to younger women, resulting in dramatic $(>70 \%)$ reductions in prescriptions for hormone therapy. 
Menopause practitioners stopped prescribing HRT. Women who were already on the HRT discontinued the therapy. And a wave of fear about HRT spread across the globe. A decade has passed since the WHI reports were published. Even though the practice of universal HRT has not resumed, HRT today has a definite role in management of menopause. This editorial will focus upon health concerns after menopause and the place of HRT today.

\section{Vasomotor Symptoms}

Hot flashes are the primary reason women seek care at menopause and request hormone therapy. Systemic estrogen therapy is the most effective treatment available for vasomotor symptoms and the associated sleep disturbance. If cessation of HRT is desired, it is important to reduce the dose slowly over several months. When a woman chooses not to take estrogen or when it is contraindicated, progestin therapy alone is an option. Medroxyprogesterone acetate [3] (MPA) and megestrol acetate [4] effectively treat vasomotor symptoms. Several drugs that alter central neurotransmitter pathways, selective serotonin reuptake inhibitors and other antidepressants, also are effective.

Today, HRT is recommended for the effective treatment of vasomotor symptoms, but only for short duration and small doses.

\section{Urogenital Atrophy}

Urinary symptoms are common complaints of menopausal women which respond well to therapy. Systemic estrogen therapy is very effective for the relief of vaginal dryness, dyspareunia, and urinary symptoms. Another option for women who should not (or choose not to) use standard estrogen therapy is topical application. Low doses of estrogen cream are effective. Lubricants are a non-hormonal alternative for reducing discomfort during intercourse when urogenital atrophy is present.

\section{Osteoporosis}

Osteoporosis is an important health concern after menopause. As therapy is most likely to benefit those at highest risk, it is important to review a woman's risk factors for osteoporosis when making treatment decisions and to consider bone mineral density screening for high-risk women. Many women have diets deficient in calcium and vitamin D and will benefit from dietary changes and supplementation. Reducing the risk of osteoporosis is another of the many health benefits of smoking cessation and regular exercise. Hormone therapy is very effective at both preventing and treating osteoporosis. The WHI [1] randomized, controlled trial confirms a significant $34 \%$ reduction in hip fractures in healthy women receiving HRT after a mean follow-up of 5 years.

Bisphosphonates, including alendronate and risedronate, specifically inhibit bone resorption and are very effective for both osteoporosis prevention and treatment. Raloxifene is a SERM which has estrogen-like actions on bone and lipids without stimulating the breast or endometrium. Calcitonin nasal spray and parathyroid hormone injections (teriparatide) are other therapies for osteoporosis.

Since options other than HRT are so effective, HRT purely for prevention and treatment of osteoporosis cannot be recommended after the WHI reports.

\section{Cardiovascular Disease (CVD)}

CVD is the leading cause of death for women, accounting for approximately $45 \%$ of mortality. Advising women to alter modifiable risk factors and adequately treating diabetes, hypertension, and hypercholesterolemia are important measures in reducing the risk of heart disease. In the past, prevention of heart disease was thought to be a potential benefit of hormone therapy use. Epidemiologic studies report an approximately $50 \%$ decrease in heart disease in women who use HRT. Observational studies are prone to bias. However, the WHI trial [1] was a randomized, controlled trial of combination hormone therapy versus placebo and showed that HRT does not prevent heart disease in healthy women, and actually increases it.

There is no role for estrogen therapy in the prevention of coronary heart disease (CHD) not only in healthy women, but also in women with established heart disease. The Heart and Estrogen/progestin Replacement Study [5], a randomized, placebo-controlled trial of HRT for secondary prevention of heart disease, also did not demonstrate any reduction in CHD events overall. The risk of cardiovascular events was actually greater in HRT users during the first year of treatment.

The latest WHI [6] has noted that younger women (50-59) taking HRT over a period of 10 years have shown no increased risk of developing CVD. This is in contrast to evidence from the previous WHI study which stated an opposite finding, linking the risk of developing CVD to commencing HRT given in the form of a combination of 
estrogens with medroxyprogesterone acetate. It may be wiser to start HRT early after menopause.

It is now established that HRT also increases the risk of stroke. This increased risk is mainly related to an increased risk of ischemic stroke. Oral estrogen alone and combined with progestogen are associated with a similar increased risk, which may be dose dependent. Conversely, a low dose of transdermal estrogens with or without a progestogen does not seem to be associated with such an increased risk of stroke, whereas the impact of tibolone, a synthetic steroid, remains uncertain. In summary, there is now a large amount of evidence demonstrating that HRT is associated with an increased risk of stroke, in particular, the ischemic subtype [7]. Hence, transdermal estrogens may be a good choice.

Raloxifene has several beneficial effects on lipids, interestingly, in a secondary analysis of results from a randomized, controlled trial of raloxifene versus placebo in more than 7,000 women with osteoporosis. There were no significant differences between groups in coronary and cerebrovascular events. Among a subset of approximately 1,000 women with increased cardiovascular risk at baseline, the women who received raloxifene experienced a significant $40 \%$ reduction in cardiovascular events [8]. But, raloxifene should not yet be prescribed to prevent cardiovascular events as it is not FDA approved for this purpose.

\section{HRT and Cancer}

There is no correlation between HRT and vulval carcinomas as well as vaginal carcinoma. The WHI results showed improvement in colorectal cancer risk with HRT. However, HRT seems to increase the risk of ovarian cancer. The recent Million Women Study [9] has revealed an increased incidence of developing ovarian cancer in women on HRT compared to women who have never used HRT. However, these risks are statistically small. The risk of developing ovarian cancer returns to pre-use levels once users stop using HRT, as per their reports. But, HRT is linked with the breast carcinoma especially when estrogen and progesterone are used for a longer period and Endometrial carcinoma when estrogen only is used.

\section{Breast Cancer}

The rising incidence of breast cancer is a matter of great health concern. The long-term use of hormone therapy is associated with an increased risk of breast cancer. Observational studies demonstrate a relative risk of approximately 1.3 with long-term HRT use, generally defined as $>5$ years. The WHI HRT trial [1] found a significant $26 \%$ increase in the risk of invasive breast cancer in women assigned to HRT after approximately 5 years of use. Interestingly, the WHI trial of estrogen alone [2] in women with prior hysterectomy demonstrated no increased risk of breast cancer after an average of 7 years of estrogen use.

A recent study found that postmenopausal women who take combined estrogen plus progestin HRT for at least 5 years are increasing their risk of breast cancer. Researchers also found that women can quickly reduce their risk of breast cancer by stopping HRT [10].

This research clearly suggests that long-term use of HRT containing medroxyprogesterone increases the risk of breast cancer, and hence should not be recommended.

Postmenopausal women receiving raloxifene as part of a large osteoporosis treatment trial experienced a $76 \%$ reduction in the risk of invasive breast cancer compared to placebo-treated women [11].

There are risks associated with SERM use. Tamoxifen and raloxifene increase the risk of VTEs approximately threefold, similar to the increased risk seen in HRT users. Hot flashes are increased with raloxifene and tamoxifen use, and raloxifene is associated with leg cramps. Tamoxifen acts as an estrogen agonist in the endometrium, increasing the risk of endometrial polyps, hyperplasia, and cancer, while no endometrial stimulation is seen with raloxifene.

Screening mammography annually for women older than the age of 50 years reduces breast cancer mortality according to several large studies. Women at an increased risk for breast cancer are advised not to use HRT or to use it only in the short term.

\section{Endometrial Cancer}

The use of unopposed estrogen is associated with an increased risk of endometrial hyperplasia and cancer. Combination estrogen-progestin therapy, therefore, is recommended for all women with a uterus. Several combination therapies may have a lower incidence of bleeding, including norethindrone acetate with either ethinyl estradiol or estradiol or low-dose conjugated equine estrogens and MPA (0.45/1.5 or 0.3/1). Women using topical estrogens should also be cautious; however, routine progesterone supplementation is not necessary.

\section{Alzheimer's Disease}

Alzheimer's disease is the most common form of dementia. Women are at a greater risk for developing the disease than 
men. A WHI Memory Study (WHIMS) [12], a randomized, double-blind, placebo-controlled trial of women ages 65 years and older enrolled in the WHI trial, showed that in contrast to the findings of observational studies, women randomized to estrogen therapy in WHIMS experienced a significant twofold increased risk of dementia.

\section{What Does Current Evidence Say?}

An updated Cochrane database review [13] included nineteen trials involving 41,904 women. In relatively healthy women, combined continuous HRT significantly increased the risk of venous thrombo-embolism or coronary event (after 1 year's use), stroke (after 3 years), breast cancer, and gallbladder disease. Long-term estrogen-only HRT significantly increased the risk of venous thromboembolism, stroke, and gallbladder disease (after 1 to 2, 3, and 7 years' use, respectively), but did not significantly increase the risk of breast cancer. The only statistically significant benefits of HRT were the decreased incidence of fractures and (for combined HRT) colon cancer with longterm use. Among women aged over 65 who were relatively healthy (i.e., generally fit without overt disease) and taking continuous combined HRT, there was a statistically significant increase in the incidence of dementia. Among women with cardiovascular disease, long-term use of combined continuous HRT significantly increased the risk of venous thrombo-embolism.

With this evidence, HRT is not indicated for the routine management of chronic disease.

Women need to be informed of the potential benefits and risks of all therapeutic options and care should be individualized based on a woman's needs and preferences. Based on the current evidence, therefore, starting HRT at the early onset of the menopause, and carrying on for a few years, apparently carries little risk in healthy women. Taking medication also confers some benefit to bone strength [13] and a small reduction in the risk of colonic cancer [14]. All women commencing HRT should be advised on type, dose, mode of delivery, and duration, and doctors should tailor treatment to individual patients. HRT is effective for preventing osteoporotic fractures, but the NAMS [15] recommends that hormone therapy for this purpose should be weighed against potential harm and other approved preventive treatments such as bisphosphonates should be considered. There are many options available to address the quality of life and health concerns of menopausal women. Currently, the primary indication for hormone therapy is the alleviation of hot flashes and associated symptoms. In the post-WHI era, HRT should be given in the lowest possible dose and for the shortest possible time to relieve symptoms. As concerns have grown regarding the risks of HRT, alternative therapies have increasingly been sought.

\section{References}

1. Writing Group for the Women's Health Initiative. Investigators risks and benefits of estrogen plus progestin in healthy postmenopausal women: principal results from the women's health initiative randomized controlled trial. JAMA. 2002;288:321-33.

2. Anderson GL, Limacher M, Assaf AR, Bassford T, Beresford SA, Black H, Bonds D, Brunner R, Brzyski R, Caan B, Chlebowski R, Curb D, Gass M, Hays J, Heiss G, Hendrix S, Howard BV, Hsia J, Hubbell A, Jackson R, Johnson KC, Judd H, Kotchen JM, Kuller L, LaCroix AZ, Lane D, Langer RD, Lasser N, Lewis CE, Manson J, Margolis K, Ockene J, O’Sullivan MJ, Phillips L, Prentice RL, Ritenbaugh C, Robbins J, Rossouw JE, Sarto G, Stefanick ML, Van Horn L, Wactawski-Wende J, Wallace R, Wassertheil-Smoller S, Women's Health Initiative Steering Committee. Effects of conjugated equine estrogen in postmenopausal women with hysterectomy: the Women's Health Initiative randomized controlled trial. JAMA. 2004;291(14):1701-12.

3. Prior JC, Nielsen JD, Hitchcock CL, et al. Medroxyprogesterone and conjugated oestrogen are equivalent for hot flushes: a 1-year randomized double-blind trial following premenopausal ovariectomy. Clin Sci (Lond). 2007;112(10):517-25.

4. Goodwin JW, Green SJ, Moinpour CM, et al. Phase III randomized placebo-controlled trial of two doses of megestrol acetate as treatment for menopausal symptoms in women with breast cancer: Southwest Oncology Group Study 9626. J Clin Oncol. 2008;26(10):1650-6.

5. Hulley S, Grady D, Bush T, Furberg C, Herrington D, Riggs B, Vittinghoff E. Randomized trial of estrogen plus progestin for secondary prevention of coronary heart disease in postmenopausal women. Heart and Estrogen/progestin Replacement Study (HERS) Research Group. JAMA. 1998;280(7):605-13.

6. Rossouw JE, Prentice RL, Manson JE, et al. Postmenopausal hormone therapy and risk of cardiovascular disease by age and years since menopause. JAMA. 2007;297(13):1465-77.

7. Renoux C, Suissa S. Hormone therapy administration in postmenopausal women and risk of stroke. Womens Health. 2011; 7(3):355-61.

8. Barrett-Connor E, Grady D, Sashegyi A, et al. MORE investigators (multiple outcomes of raloxifene evaluation). Raloxifene and cardiovascular events in osteoporotic postmenopausal women: four-year results from the MORE (multiple outcomes of raloxifene evaluation) randomized trial. JAMA. 2002;287(7): 847-57.

9. Beral V, Million Women Study Collaborators, Bull D, Green J, et al. Ovarian cancer and hormone replacement therapy in the million women study. Lancet. 2007;369(9574):1703-10.

10. Chlebowski RT, Kuller LH, Prentice RL, et al. WHI investigators breast cancer after use of estrogen plus progestin in postmenopausal women. N Engl J Med. 2009;360(6):573-87.

11. Recker RR, Mitlak BH, Ni X, et al. Long-term raloxifene for postmenopausal osteoporosis. Curr Med Res Opin. 2011;27(9): 1755-61.

12. Shumaker SA, Legault C, Kuller L, et al. Women's Health Initiative Memory Study. Conjugated equine estrogens and incidence of probable dementia and mild cognitive impairment in postmenopausal women: Women's Health Initiative Memory Study. JAMA. 2004;291(24):2947-58. 
13. Farquhar C, Marjoribanks J, Lethaby A, et al. Long term hormone therapy for perimenopausal and postmenopausal women. Cochrane Database Syst Rev. 2009;15(2): CD004143.

14. Johnson JR, Lacey JV Jr, Lazovich D, et al. Menopausal hormone therapy and risk of colorectal cancer. Cancer Epidemiol Biomark Prev. 2009; 18(1):196-203.
15. Estrogen and progestogen use in peri- and postmenopausal women: March 2007 position statement of the North American Menopause Society. Menopause 2007;14:1-17. 